\title{
Denoising of ECG Signal by using Adaptive Filter and Non Adaptive Filter
}

\author{
Abhishek Sahu', Sagar Singh Rathore ${ }^{2}$ \\ M.E Scholar, Department of Electronics and Telecommunication, SSTC (BHILAI) ${ }^{1}$ \\ Asst. Professor, Department of Electronics and Instrumentation, SSTC (BHILAI) ${ }^{2}$
}

\begin{abstract}
Electrocardiogram (ECG) is an effective non-invasive method used to detect cardiac abnormalities. In our paper, we provide a study various noises, example power line disturbance (PLI), movement artifacts, electrode touch noise, muscle relaxation, base line drift, electromyography noise (EMG) and instrumentation noise etc. To remove above noises various algorithms of different filter, non-adaptive filter are used and we also provide discrete wavelet transform DWT. To filter random artifacts, filter with constant parameters, because hum manner is not accurate known relevant on time. For this problem to solve digital filter are used such as adaptive filters as smallest (least) mean square (LMS), Normalized mean square error (NLMS), Recursive least square (RLS), sign LMS, sign-sign LMS algorithms In the comparison among all have been tabulated. The quality of algorithms are evaluated by signal to noise ratio (SNR), mean square error (MSE), rate root mean difference (\%PRD) and standardized mean square (NMSE). In the comparison to various adaptive algorithms sign-sign LMS gives better result for all parameters with MSE $=0.0253$, $\mathrm{NRMSE}=0.0033, \% \mathrm{PRD}=0.3231, \mathrm{SNR}=5.327$.
\end{abstract}

Keywords: ECG, LMS, NLMS, RLS, SLMS, SNR, MSE, NMSE, \%PRD.

\section{INTRODUCTION}

The signal of ECG is basic devices for the analysis and finding different cardiovascular issues. At the time when ECG signal is recorded, it might be debased by many types of noises such as power line disturbance, base line wandering, motion artifacts, electrode touch noise, muscle contraction, instrumentation, and electrosurgical noise etc [1]. The power line disturbance causes interference due to frequency of $50 \mathrm{~Hz}$, because present in the ECG signal band (0.05$100 \mathrm{~Hz}$ ). This noise corrupt the ST segment poor the quality of signal, frequency resolution and gives higher amplitude signals in ECG signal that conceal the features which are important for clinical approach and diagnosis. Filter of these noises in ECG signal is basic work for best diagnosis. The filter of more resolution ECG signal from undesired ECG signal which are present with hidden noise is an basic work of research. The ECG signal enhancement is to filter the required signal component from the undesired artifacts. So as to introduce an ECG that gives ECG and accurate interpretation. So Many methods have been presented in the literature to provide ECG enhancement using adaptive methods [1-13], adaptive filtering methods help to determine time changes voltages and to track the changes of signals. In [3], Thakor et al. Proposed an least mean square based adaptive filter to acquire the impulse response of normal QRS complexes and then approach it for arrhythmia detection in corrupted ECG recordings. In the LMS algorithm works on an instant basis such that weight vector is renewed for each sample. The calculation complexity can be decrease by providing the sign based algorithms, such as, the signed regressor algorithm, the sign algorithm and the sign-sign algorithm $[14,15]$. In order to work with both the difficulty and convergence issues we used various adaptive filter structure based on standardized sign regressor LMS (NSRLMS) algorithm, normalized sign LMS (NLMS) algorithm and normalized sign-sign least mean square error (NSSLMS) algorithm. These algorithms find minimum arithmetic difficulty because of the sign present in the algorithm and better filtering because of the normalized terms [16, 17]. Adaptive algorithms are compared with the performance evaluators such as signal to noise ratio (SNR), mean square error (MSE), normalized mean square error(NMSE), percentage root square difference (\%PRD), after compare we find sign-sign LMS gives better result.

\section{MATERIALS AND METHODS}

\subsection{ECG RECORDING}

MIT-BIH arrhythmia database [17] was also use to recording ECG signal. It consist of 48 annotated records, obtain from 46 subjects studied by the arrhythmia lab of bath Israel hospital in Boston between 1975 and 1979 the database contain several record in the 100 series were taken for our research purpose every record in the arrhythmia database is slightly over $30 \mathrm{~min}$. In size having sampling frequency $360 \mathrm{~Hz}$. Header files include information about leads used the patient's age, sex and medications [18, 19]. 


\subsection{VARIOUS TYPES OF NOISES:}

\subsubsection{Power line interference}

Power line interference (PLI) noise occurs due to two coupling such as capacitive coupling and inductive coupling.[20] Capacitive coupling refers to the transfer of energy between two circuits by means of coupling capacitance present in the two circuits. Inductive coupling is caused due to mutual inductance between two conductors. Capacitive coupling and inductive coupling responsible for high frequency and low frequency noise respectively. Inductive coupling is more dominant for power line interference in ECG. The power line disturbance noise is causes due to $50 \mathrm{~Hz}$ or $60 \mathrm{~Hz}$ depending on the power supply. [16]

1.2.2. Electrode contact noise

It is caused due to changes in the position of the heart with respect to the electrodes and changes in the propagation medium between the electrode position and heart. This causes variations in the amplitude of the ECG signal, as well as frequency baseline shifts. poorer conductivity between the electrodes and the skin decrease the strength of signal. [18] 1.2.3. Motion Artifacts

Motion artifacts are due to changes of baseline cause by electrode motion. The main cause of movement artifacts are vibrations, movements etc. In this ECG signal the baseline drift occurs at low frequency (less than $1 \mathrm{~Hz}$ ). Motion artifacts depend on the electrode properties and electrolyte properties. [20]

1.2.4. Electromyography Noise (EMG)

EMG noise is causes due to the contraction of muscles besides the heart. EMG noise is randomly in nature and designed by Gaussian distribution function. The mean of this noise is assumed to be zero and variance depends on the environmental changes. Frequency of EMG noise is in between 100-500 Hz. [20]

1.2.5. Instrumentation noises

Noises also introduced due to measuring instrument, major source of such noise are electrical probes, cables, signal amplifier and analog to digital converter. Another types of noise is colour noise or flicker noise is low frequency electronics noise. [18]

1.2. Adaptive Filter

Adaptive filter works as a linear filter, transfer function of adaptive filter is controlled by changeable parameters (coefficient). Its numerous functions are to adjust that variable parameter according to optimization algorithm and adapt to change in signal characteristics in order to reduce the error. It involves variation of filter coefficients over time. The vector representation of input signal [23] $\mathrm{X}(\mathrm{n})$ is $\mathrm{X}(\mathrm{n})=[\mathrm{x}(\mathrm{n}), \mathrm{x}(\mathrm{n}-1),-, \mathrm{x}(\mathrm{n}-\mathrm{N}-1)]$

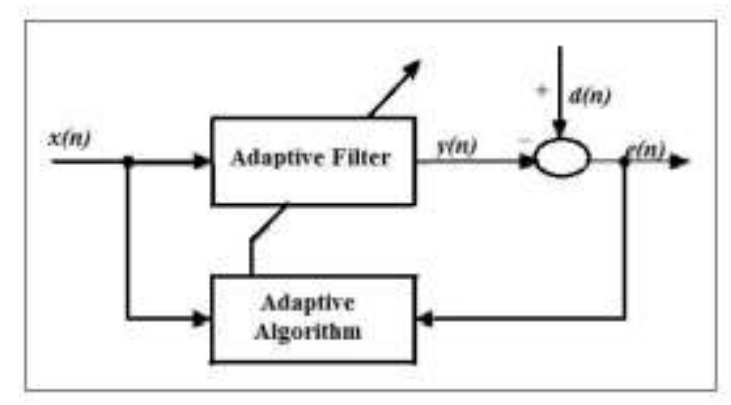

Figure 1 Block Diagram of Adaptive Filter [12]

Signal apply at input of adaptive filter is corrupted with noises. It becomes sum of desired signal $d(n)$ and noise e(n). Adaptive filter has finite impulse response (FIR) structure. Its impulse response is equal to filter coefficient. The coefficients for a filter of order $\mathrm{N}[19]$.

$$
\mathrm{W}(\mathrm{n})=[\mathrm{w}(0), \mathrm{w}(1),---, \mathrm{w}(\mathrm{N}-1)] \mathrm{T}
$$

Output of adaptive filter is $y(n)$

$$
\mathrm{y}(\mathrm{n})=\mathrm{W}(\mathrm{n}) \mathrm{T} X(\mathrm{n})
$$

Error signal is

$$
E(n)=d(n)-y(n)
$$

At every time instant variable filter update the filter coefficients

$$
\mathrm{W}(\mathrm{n}+1)=\mathrm{W}(\mathrm{n})+\Delta \mathrm{W}(\mathrm{n})
$$

Where $\Delta \mathrm{W}(\mathrm{n})$ is correction factor for filter coefficients

\subsection{1- LMS (least mean square)}

It works only on error at the current time, hence filter weights are only adapted based on the error at the current time [20].

According to this algorithm updated weight is given by 
$\mathrm{W}(\mathrm{n}+1)=\mathrm{W}(\mathrm{n})+2 \cdot \mu \cdot \mathrm{X}(\mathrm{n}) \cdot \mathrm{e}(\mathrm{n})$

Where. $\mu$ is step size.

\subsection{2-NLMS (Normalized mean square)}

It is upgraded version of LMS; update the coefficients of adaptive filter. Step size of NLMS algorithm is time varying.[20]

According to this algorithm the updated weight is given by

$$
W(n+1)=w(n)+2 \cdot \mu\left[\frac{x(n)}{\bmod \text { of } x(n) \cdot 2}\right] \cdot e(n)(7)
$$

Where $\mu(n)=\mu /[\bmod$ of $x(n) 2]$

\subsection{3-SELMS algorithm-}

In this algorithm sign function is applied to error signal to update the filter coefficient of adaptive filter.[19] According to this algorithm the updated weight is given by

$\mathrm{W}(\mathrm{n}+1)=\mathrm{W}(\mathrm{n})+2 \cdot \mu \mathrm{x}(\mathrm{n}) \cdot \operatorname{sgn}(\mathrm{e}(\mathrm{n}))$

\subsection{4- SSLMS algorithm-}

In this algorithm sign function is applied to both input and error signal to update the filter coefficient of adaptive filter. [20]

According to this algorithm the updated weight is given by

$$
W(n+1)=w(n)+2 \cdot \mu \cdot \operatorname{sgn}(x(n) \cdot \operatorname{sign}(e(n))
$$

\section{Discrete Wavelet Transform (DWT)}

For analysis of non-stationary signals wavelet transform is a powerful method. ECG signal are non-stationary and time varying signals. So wavelet transform is suitable for Analysis of ECG signal. Wavelet allow both time and frequency analysis of signals. Discrete Wavelet transform analyse and a signal as a linear combination of the sum of the product of Wavelet coefficients.[20]

\section{PERFORMANCE EVALUATION PARAMETERS}

Time Domain Analysis:

If $\mathrm{x}(\mathrm{n})$ is the recorded signal or ECG signal, $\mathrm{xn}$, represents the noisy signal and $\mathrm{xm}$ is the filtered ECG signal, mean square error (MSE) is defined as [20].

$M S E=\frac{1}{N} \sum_{n=0}^{N-1}[x(n)-x m(n)] \wedge 2$

The Normalized form of NMSE is

$N M S E=\frac{\sum_{n=0}^{N-1}[x(n)-x m(n)]^{\wedge} 2}{\sum_{n=0}^{N-1}[x(n)]^{\wedge} 2}$

The Normalized form of RMSE

$N R M S E=\left[\frac{\sum_{n=0}^{N-1}[x(n)-x m(n)]^{\wedge} 2}{\sum_{n=0}^{N-1}[x(n)]^{\wedge} 2}\right] \wedge \frac{1}{2}$

Percentage root mean square error difference (\%PRD)

$\% \mathrm{PRD}=\left[\frac{\sum_{\mathrm{n}=0}^{\mathrm{N}-1}[\mathrm{x}(\mathrm{n})-\mathrm{xm}(\mathrm{n})]^{\wedge} 2}{\sum_{\mathrm{n}=0}^{\mathrm{N}=0}[\mathrm{x}(\mathrm{n})]^{\wedge} 2}\right] \wedge \frac{1}{2} * 100 \%$

Signal to Noise Ratio in $\mathrm{dB}$ (SNR)

$\operatorname{SNR}[\mathrm{dB}]=10 \log \left[\frac{\sum|\mathrm{xn}-\mathrm{x}(\mathrm{n})| \wedge 2}{\sum|\mathrm{xm}-\mathrm{x}(\mathrm{n})| \wedge 2}\right]$

\section{RESULT}

The values of various performance evaluators (SNR, \%PRD, MSE, NRMSE, RMSE) of noisy (colour noise), adaptive RLS filtered and adaptive NLMS filtered, adaptive LMS filtered, adaptive sign-LMS filtered and adaptive QDRLS filtered ECG signal are evaluated shown in Table 1. We know that the high values of SNR and low values of \%PRD and MSE are good. From Table 1 we find that noisy signal's SNR is very low, but all filtered signal have high SNR after filtering signal have low \%PRD and MSE. For noisy ECG signal (Gaussian noise) are filtered using various adaptive algorithms and parameters shown in Table 2. Similarly Table 3 shows result for noisy ECG signal due to power line interference noise .In the result, Fig.1,Fig.2, Fig.3, Fig.4, Fig.5, Fig.6 shows Gaussian noise added in ECG signal and filtered using NLMS, RLS, S-LMS, LMS, QDRLS algorithms respectively. After study the result, we find 
out which is the better adaptive filter for removal of ECG noise and showing better result for the performance parameters. In Fig.7, Fig.8, Fig. 9, Fig.10, Fig. 11, shows colour noise added with ECG signal and filtered using various adaptive algorithms such as NLMS, RLS, SS-LMS, S-LMS, QDRLS, respectively. Similarly, Fig.12, Fig.13, Fig. 14, Fig. 15, shows for the power noise. Table 1 shows that comparison of different algorithms using performance parameters for colour noise. Table 2 shows that comparison of different algorithms using performance parameters for Gaussian noise. similarly Table 3 shows that performance evaluation for power line interference noise.
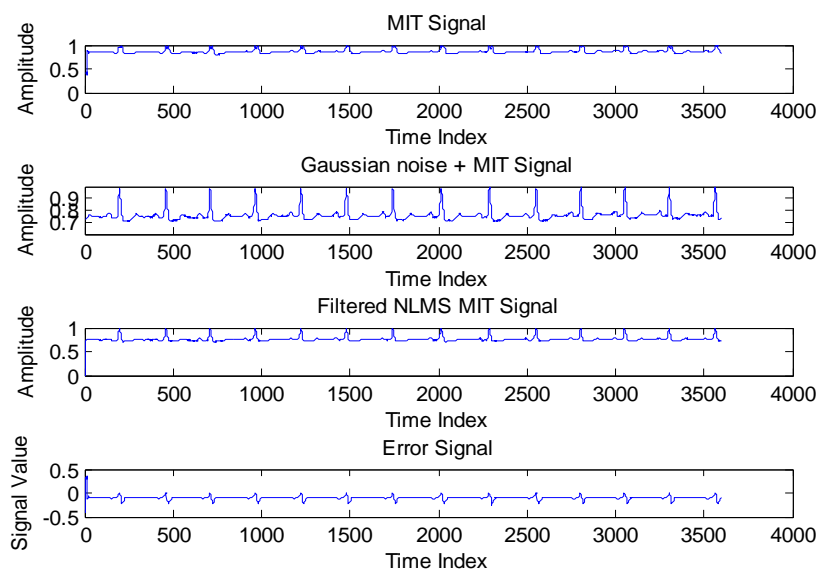

Fig.1 Gaussian noise added with ECG signal and filtered filtered NLMS algorithms
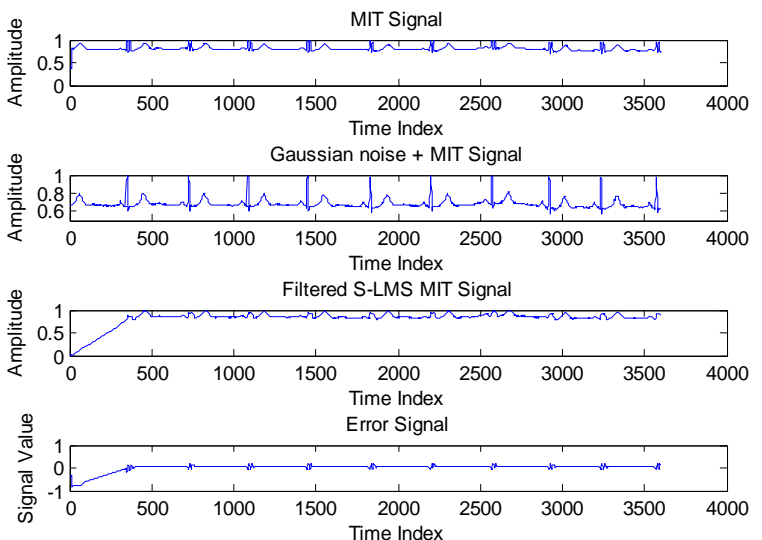

Fig. 3 Gaussian noise added with ECG signal and filtered
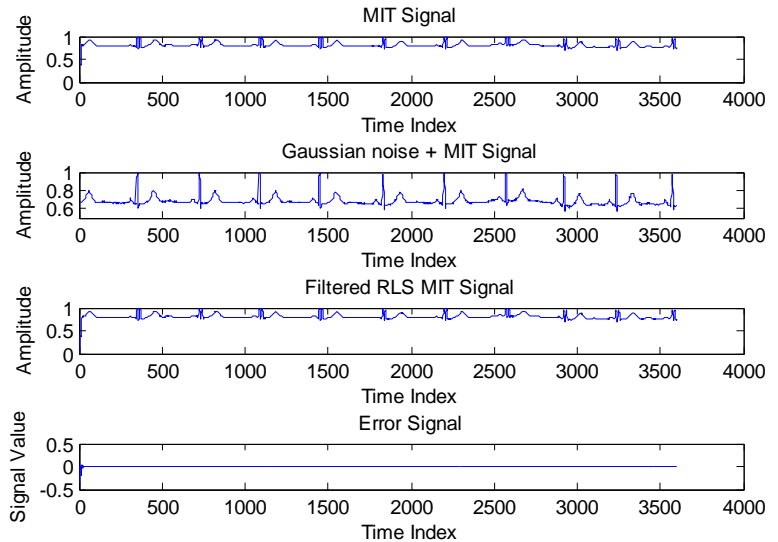

Fig. 2 Gaussian noise added with ECG signal and RLS algorithms
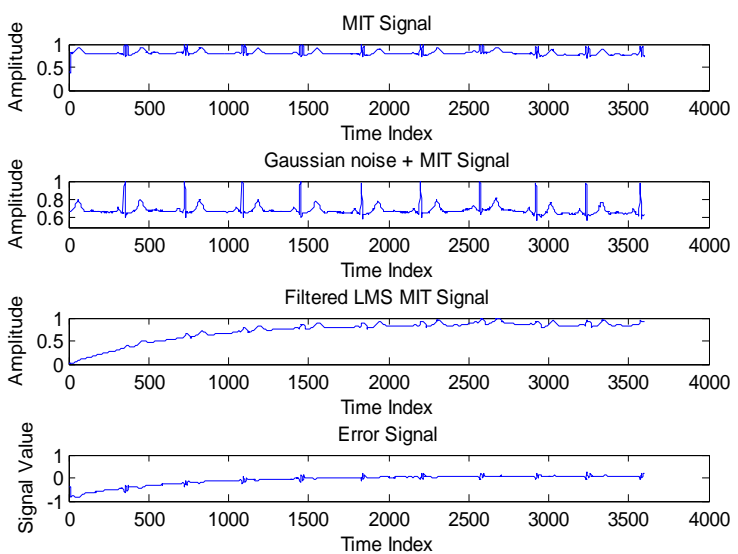

Fig.4 Gaussian noise filtered using LMS algorithms lgorithms
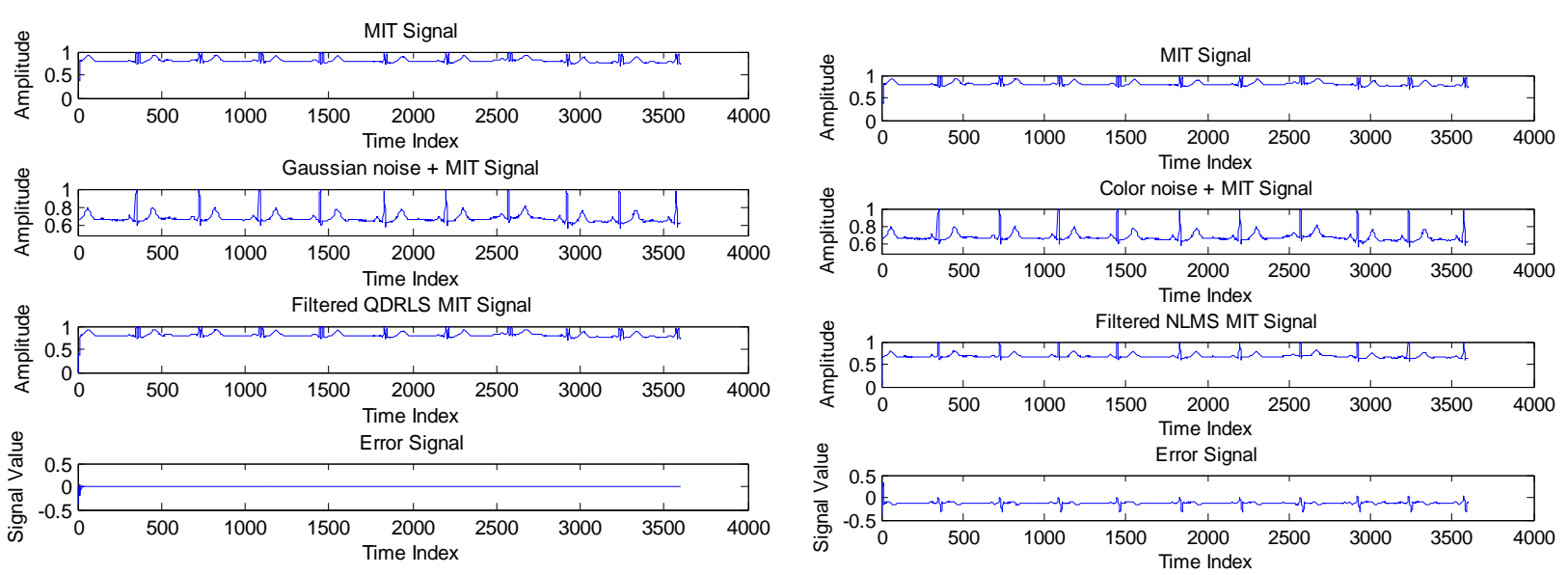

Fig. 5 Gaussian noise filtered using QDRLS algorithms

Fig. 6 colour noise Filtered using NLMS algorithm 

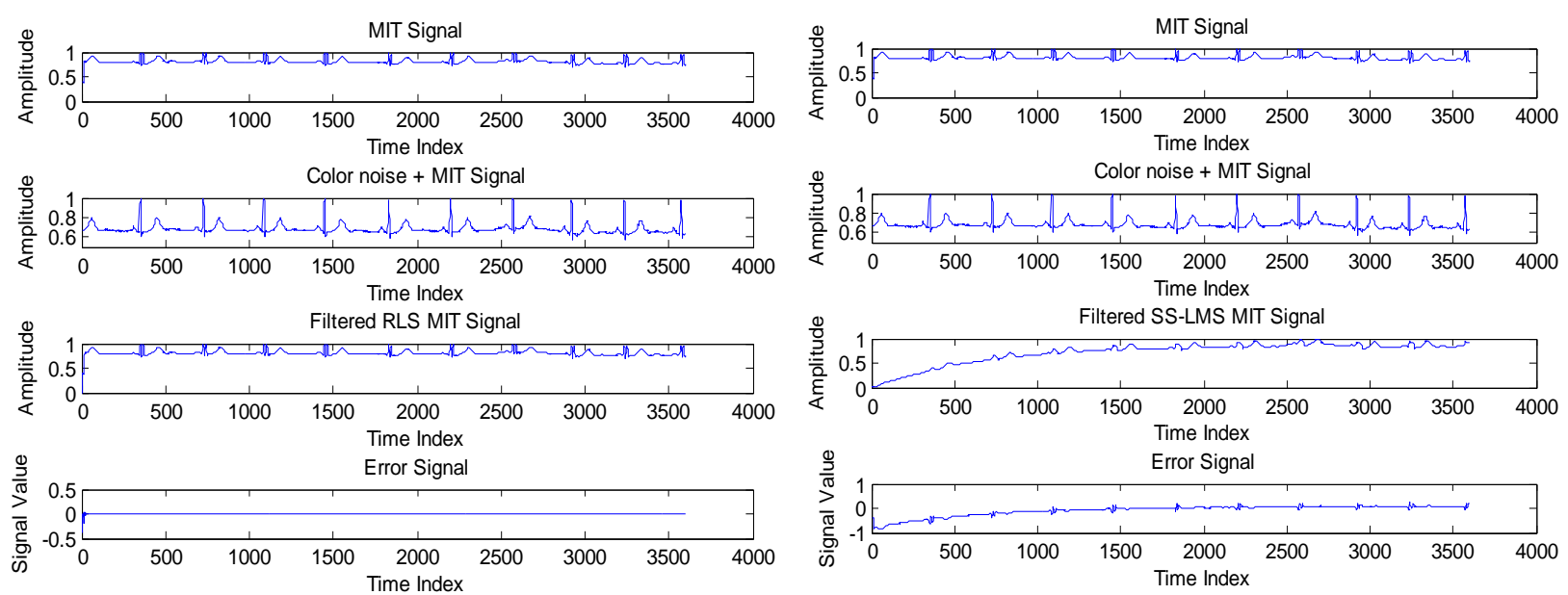

Fig.7 colour noise filtered using RLS algorithms

Fig. 8 colour noise filtered using SSLMS algorithms
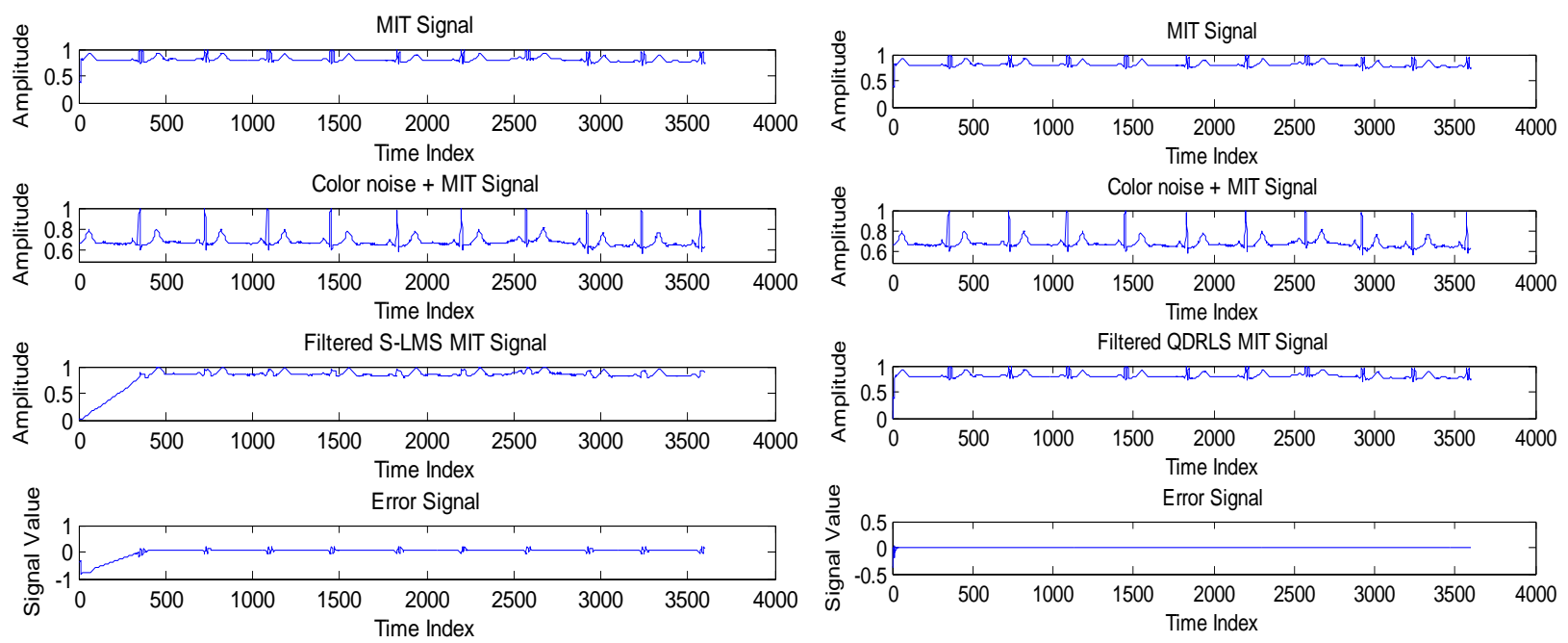

Fig .9 colour noise filtered using SLMS algorithms

Fig.10 colour noise filtered using QDRLS algorithm
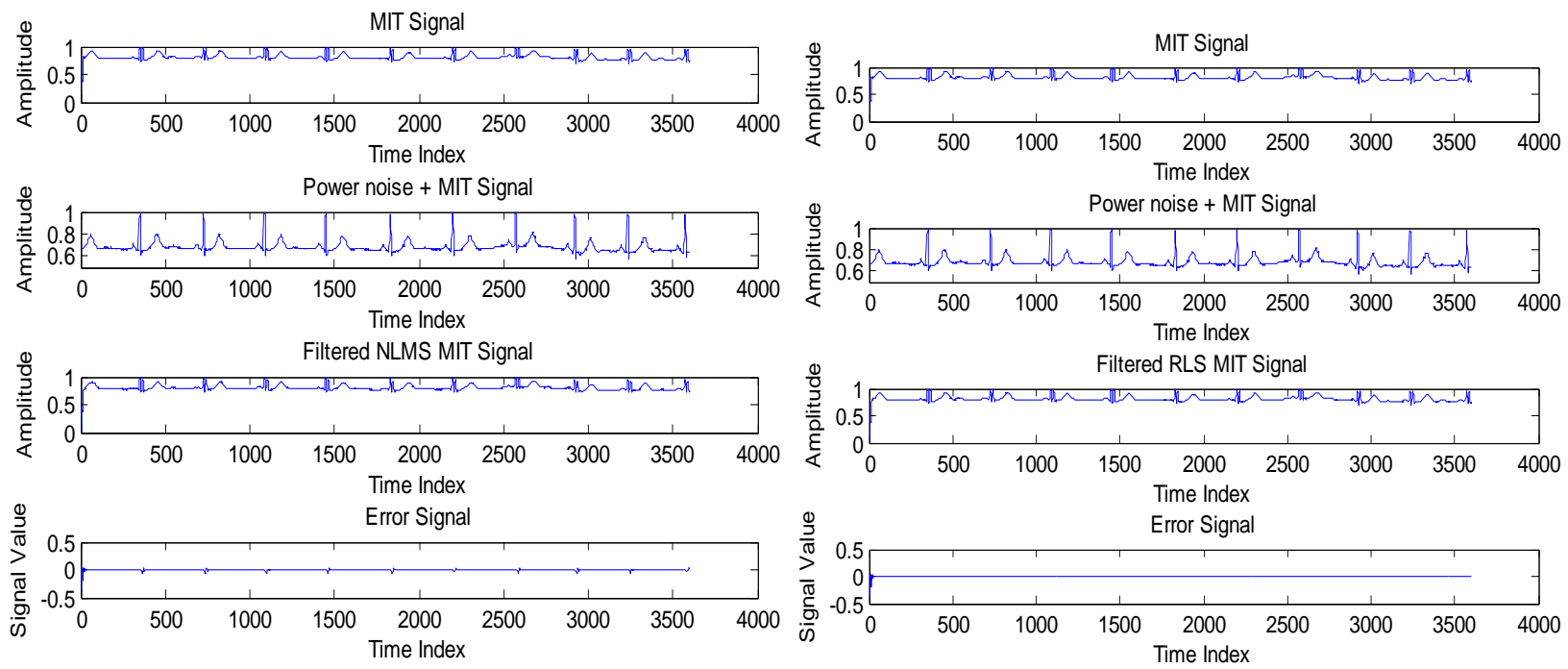

Fig. 11 power noise filtered using NLMS algorithms

Fig. 12 power noise filtered using RLS algorithms 

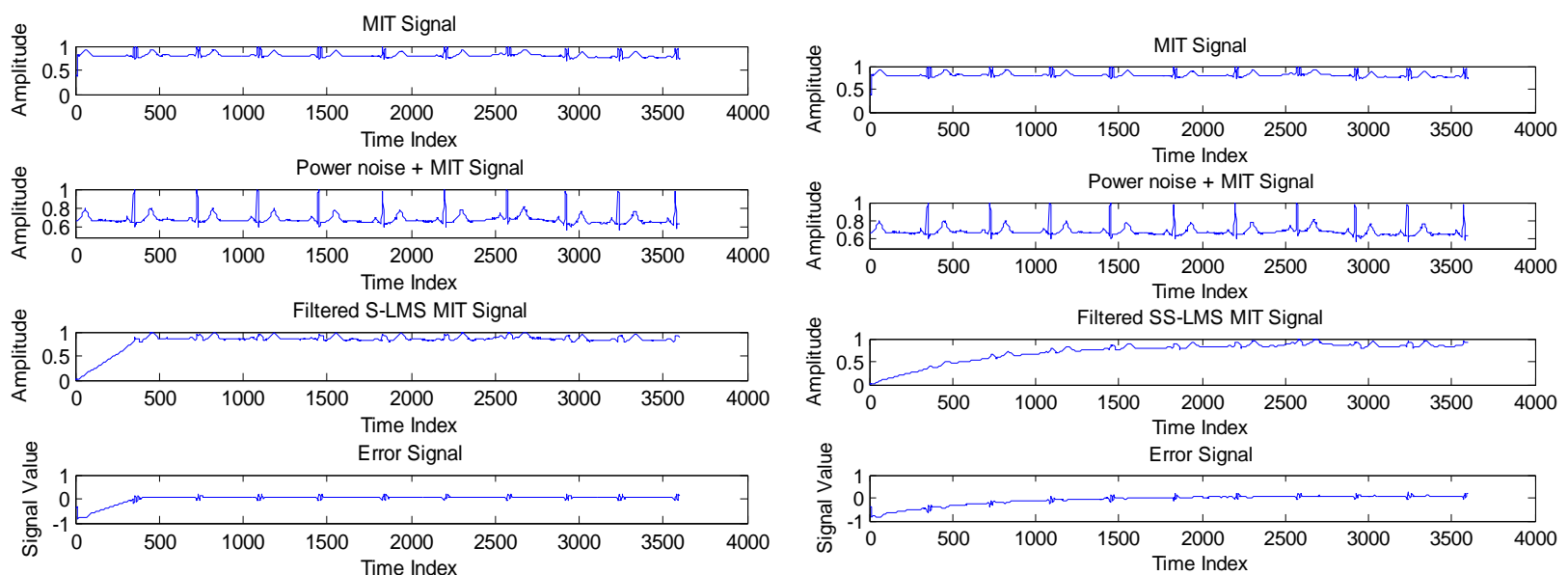

Fig. 14 power noise filtered using SSLMS algorithms

Fig. 13 power noise filtered using SLMS algorithms
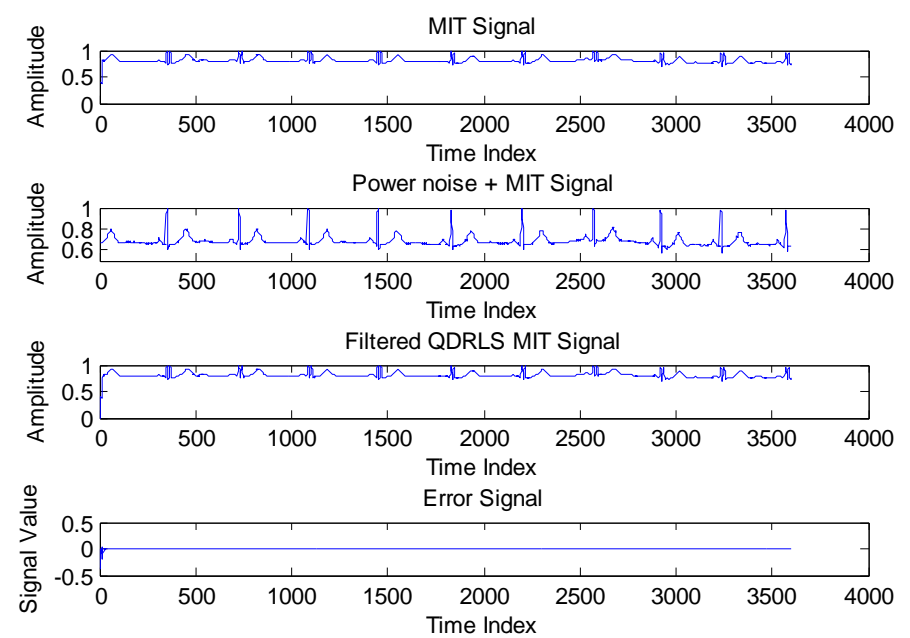

Fig.15 power noise filtered using QDRLS algorithms

Table 1 Comparison of different algorithms using performance parameters for colour noise

\begin{tabular}{|l|l|l|l|l|l|}
\hline \multirow{2}{*}{ PARAMETERS } & \multicolumn{5}{|c|}{ PERFORMANCE EVALUATORS OF COLOR NOISE } \\
\cline { 2 - 6 } & \multicolumn{5}{|c|}{ ECG RECORDED DATA 105 DATABASE } \\
\hline & MSE & NRMSE & \%PRD & RMSE & SNR \\
\hline NLMS & 0.0178 & 0.0027 & 0.2620 & 0.1302 & 18.6280 \\
\hline RLS & $6.382 \mathrm{e}-05$ & $1.634 \mathrm{e}-04$ & 0.0154 & 0.0134 & -0.0300 \\
\hline SSLMS & 0.0252 & 0.0032 & 0.3231 & 0.321 & -4.3904 \\
\hline SLMS & 0.0547 & 0.0049 & 0.4752 & 0.4752 & -4.9530 \\
\hline QDRLS & $6.417 \mathrm{e}-05$ & $1.638 \mathrm{e}-04$ & 0.0172 & 0.0165 & -0.0315 \\
\hline
\end{tabular}

Table 2 Comparison of different algorithms using performance parameters for Gaussian noise

\begin{tabular}{|l|l|l|l|l|l|}
\hline \multirow{2}{*}{ PARAMETERS } & \multicolumn{5}{|c|}{ PERFORMANCE EVALUATORS OF GAUSSIAN NOISE } \\
\cline { 2 - 6 } & NSE & NRMSE & \%PRD & RMSE & SNR \\
\hline & 0.01689 & 0.0026 & 0.2671 & 0.1303 & 20.3574 \\
\hline NLMS & $6.381 \mathrm{e}-05$ & $1.634 \mathrm{e}-04$ & 0.0154 & 0.0081 & -0.0318 \\
\hline RLS & 0.02613 & 0.0032 & 0.3301 & 0.1520 & -4.3809 \\
\hline SSLMS & 0.0546 & 0.0039 & 0.4842 & 0.2349 & -4.8715 \\
\hline LMS & $6.957 \mathrm{e}-05$ & $1.716 \mathrm{e}-04$ & 0.0172 & 0.0073 & -0.0229 \\
\hline QDRLS & \multicolumn{5}{|c|}{} \\
\hline
\end{tabular}


Table 3 Comparison of different algorithms using performance parameters for power line noise

\begin{tabular}{|l|l|l|l|l|l|}
\hline \multirow{2}{*}{ PARAMETERS } & \multicolumn{5}{c|}{ PERFORMANCE EVALUATORS OF POWERLINE NOISE } \\
\cline { 2 - 6 } & \multicolumn{4}{|c|}{ ECG RECORDED DATA 106 DATABASE } \\
\hline & MSE & NRMSE & \%PRD & RMSE & SNR \\
\hline NLMS & $1.405 \mathrm{e}-04$ & $2.437 \mathrm{e}-04$ & 0.0234 & 0.0109 & 0.3735 \\
\hline RLS & $6.304 \mathrm{e}-04$ & $1.644 \mathrm{e}-04$ & 0.0155 & 0.0070 & -0.0262 \\
\hline \multicolumn{1}{|c|}{ SSLMS } & 0.0261 & 0.0032 & 0.3321 & 0.1520 & -4.3729 \\
\hline SLMS & 0.0547 & 0.0039 & 0.4752 & 0.2260 & -4.8273 \\
\hline QDRLS & $6.876 \mathrm{e}-05$ & $1.716 \mathrm{e}-04$ & 0.0162 & 0.0083 & -0.0388 \\
\hline
\end{tabular}

\section{DISCUSSION AND CONCLUSION}

In this work, we have implemented various algorithm of adaptive filter for removing artifacts contaminated with ECG signal during recording. The adaptive algorithms are LMS, NLMS, RLS, Sign-LMS, SSLMS were capable to remove noises such as white noise, colour noise, muscle artifacts, electrode contact noise, baseline wander noise, composite noise and power line interference properly. The performance of all algorithms was evaluated by parameters such as SNR, \%PRD, MSE, NMSE. In the comparison to various adaptive algorithms SSLMS gives better result for all parameters with $\mathrm{MSE}=0.0262, \mathrm{NRMSE}=0.0033, \% \mathrm{PRD}=0.3331, \mathrm{RMSE}=0.331, \mathrm{SNR}=-4.3914$.

\section{REFERENCES}

[1] E. T. Gar, C. Thomas and M. Friesen, "Comparison of Noise Sensitivity of QRS Detection Algorithms," IEEE Tran. Biomed. Eng., vol. 37 , no.1,pp. 85-98, January 1990

[2] Y. Der Lin, Y. Hen Hu, Power-line interference detection and suppression in ECG signal processing, IEEE Transactions on Biomedical Engineering 55 (January) (2008) 354-357.

[3] N.V. Thakor, Y.S. Zhu, Applications of adaptive filtering to ECG analysis: noise cancellation and arrhythmia detection, IEEE Trans- actions on Biomedical Engineering 38 (8) (1991) 785-794.

[4] J.A. Van Alste, T.S. Schilder, Removal of base-line wander and power-line interference from the ECG by an efficient FIR filter with a reduced number of taps, IEEE Transactions on Biomedical Engineering 32 (12) (1985) 1052-1060.

[5] A.K. Ziarani, A. Konrad, A nonlinear adaptive method of elimination of power line interference in ECG signals, IEEE Transactions on Biomedical Engineering 49 (6) (2002) 540-547.

[6] S. Olmos, J. Garcia, R. Jane, P. Laguna, ECG signal compression plus noise filtering with truncated orthogonal expansions, Signal Processing 79 (1999) $97-115$.

[7] J.M. Leski, N. Henzel, ECG baseline wander and power line interference reduction using nonlinear filter bank, Signal Processing 85 (2005) 781-793.

[8] V. Almenar, A. Albiol, A new adaptive scheme for ECG enhancement, Signal Processing 75 (November) (1999) 253-263.

[9] C. Meyer, J.F. Gavela, M. Harris, Combining algorithms in automatic detection of QRS complexes in ECG signals, IEEE Transactions on Information Technology in Biomedicine 10 (3) (2006) 468-475.

[10] C. Brouse, G.A. Bumont, F.J. Herrmann, J.M. Ansermino, A wavelet approach to detecting electrocautery noise in the ECG, IEEE Engineering in Medicine and Biology Magazine 25 (4) (2006) 76-82.

[11] C. Vaz, N.V. Thakor, Adaptive Fourier estimation of time-varying evoked potentials, IEEE Transactions on Biomedical Engineering 36 (April) (1989) 448-455.

[12] N.V. Thakor, C. Vaz, R.W. Mc Pherson, D.F. Hanley, Adaptive Fourier series modeling of time-varying evoked potentials: Electroencephalography Clinical Neurophysiology 80 (2) (1991) 108-118.

[13] A.K. Barros, M. Yoshizawa, Y. Yasuda, Filtering noncorrelated noise in impedance cardiography, IEEE Transactions on Biomedical Engineering 42 (March) (1995) 324-327.

[14] P. Laguna, R. Jane', S. Olmos, N.V. Thakor, H. Rix, P. Caminal, Adaptive estimation of QRS complex by the Hermite model for classification and ectopic beat detection, Medical \& Biological Engineering \& Computing 34 (1) (1996) 58-68.

[15] B. Farhang-Boroujeny, Adaptive Filters-Theory and Applications, John Wiley and Sons, Chichester, UK, 1998.

[16] S. Koike, Analysis of adaptive filters using normalized signed regressor LMS algorithm, IEEE Transactions on Signal Processing 47 (10) (1999) 2710-2723.

[17] E. Eweda, Analysis and design of a signed regressor LMS algorithm for stationary and nonstationary adaptive filtering with correlated Gaussian data, IEEE Transactions on Circuits and Systems 37 (11) (1990) 1367-1374.

[18] de Laboratóio M. Biopac Student Lab. Biopac Systems Inc.; 2008.

[19] Moody GB, Mark RG. The MIT-BIH arrhythmia database on CD-ROM and software for use with it. Proc. 1990 Computers in Cardiology. IEEE; 1990. pp. 185-8.

[20] Manikandan MS, Dandapat S. Wavelet energy based diagnostic distortion measure for ECG. Biomed Signal Process Control 2007;2:80-96. 